\title{
A novel hydrodynamic approach of drag-reducing polymers to improve left ventricular hypertrophy and aortic remodeling in spontaneously hypertensive rats
}

\author{
This article was published in the following Dove Press journal: \\ International Journal of Nanomedicine \\ 13 December 2016 \\ Number of times this article has been viewed
}

\author{
Xinlu Zhang,, * Xu Wang, ${ }^{2, *}$ \\ Feng Hu,' Boda Zhou, ${ }^{3}$ Hai-Bin \\ Chen,' Daogang Zha,' Yili Liu,' \\ Yansong Guo, ${ }^{4}$ Lemin Zheng, ${ }^{2}$ \\ Jiancheng Xiu' \\ 'Department of Cardiology, Nanfang \\ Hospital, Southern Medical University, \\ Guangzhou, ${ }^{2}$ The Institute of Cardiovascular \\ Sciences and Institute of Systems \\ Biomedicine, School of Basic Medical \\ Sciences, and Key Laboratory of Molecular \\ Cardiovascular Sciences of Ministry of \\ Education, Peking University Health Science \\ Center, ${ }^{3}$ Department of Cardiology, Key \\ Laboratory of Molecular Cardiovascular \\ Sciences of Ministry of Education, and Key \\ Laboratory of Cardiovascular Molecular \\ Biology and Regulatory Peptides of Ministry \\ of Health, Peking University Third Hospital, \\ Beijing, ${ }^{4}$ Department of Cardiovascular \\ Medicine, Fujian Provincial Hospital, Fuzhou, \\ People's Republic of China \\ *These authors contributed equally \\ to this work
}

Correspondence: Jiancheng Xiu

Department of Cardiology, Nanfang Hospital,

Southern Medical University, No 1838 Guang

zhou da dao bei, Baiyun District, Guangzhou

5I05 I5, People's Republic of China

Tel +86 2062787389

Fax +86 2061360416

Email xiujc@I26.com

Lemin Zheng

The Institute of Cardiovascular Sciences and Institute of Systems Biomedicine, School of Basic Medical Sciences, and Key Laboratory of Molecular Cardiovascular Sciences of Ministry of Education, Peking University Health Science Center, No 38 Xueyuan Road, Haidian District, Beijing I00191, People's Republic of China Tel +86 1082805452

Fax +86 I0 82802769

Email zhengl@bjmu.edu.cn

\begin{abstract}
Drag-reducing polymers (DRPs), when added in minute concentrations, have been shown to decrease peripheral vascular resistance. In this study, the effect of DRPs on the hypertension-induced left ventricular hypertrophy and aortic remodeling was evaluated in spontaneously hypertensive rats (SHR). Male SHR and age-matched Wistar rats were divided into four groups and received intravenous injection of normal saline (NS) or DRPs. Body weight (BW), heart rate (HR) and systolic blood pressure (SBP) were measured. Echocardiography was used to evaluate the changes in left ventricle (LV) function and global wall motion. The LV and aorta were stained by hematoxylin and eosin. Cell size of cardiomyocytes and aortic medial thickness were evaluated for each section. The expression of endothelin-1 (ET-1) of LV and aorta was examined by quantitative reverse transcription polymerase chain reaction (qRT-PCR) and immunohistochemistry. There was no significant difference in the increase of SBP among SHR + NS, SHR + 10DRP and SHR + 20DRP groups. SHR + NS group had markedly smaller left ventricular end-systolic diameter and left ventricular end-diastolic diameter but bigger anterior and posterior systolic wall thicknesses, while there was no significant difference in fractional shortening and ejection fraction. The cross-sectional areas (CSAs) of cardiomyocytes and the medial thickness of the aorta in SHR + 10 (ppm) DRP and SHR + 20 (ppm) DRP groups were significantly reduced compared with SHR + NS group. The expression of ET-1 in SHR + 10DRP and SHR + 20DRP groups was significantly attenuated. These results suggest that chronic treatment with DRPs can protect against left ventricular hypertrophy and aortic remodeling. DRPs may offer a new approach to the treatment of left ventricular hypertrophy and aortic remodeling caused by hypertension.
\end{abstract}

Keywords: aortic remodeling, drag-reducing polymers, ET-1, hypertension, left ventricular hypertrophy

\section{Introduction}

In both human and animal models, hypertension is associated with cardiac and aortic remodeling. ${ }^{1-4}$ Cardiac remodeling is defined as genome expression resulting in molecular, cellular and interstitial changes and manifested clinically as myocardial hypertrophy, fibrosis and cardiac decompensation. ${ }^{1,5}$ Aortic remodeling is characterized by impaired endothelium-dependent vasodilation and structural vascular changes, ${ }^{6}$ thus, endothelium plays a primary role in the modulation of vascular tone and structure. ${ }^{7}$ Previous research showed that both cardiac and aortic remodeling can be exacerbated by endothelin-1 (ET-1) overactivation. ${ }^{8}$ 
The process of cardiac remodeling has been found to be influenced by hemodynamic load ${ }^{9}$ and neurohormonal activation; unfortunately, the exact mechanism has not been fully elucidated. Vascular endothelium directly contacts flowing blood and transduces hemodynamic stimuli into changes in endothelial structure and function. ${ }^{10}$ Hemodynamic shear stress from blood flow regulates vascular morphogenesis and blood pressure through the endothelium. ${ }^{11}$ Recently, a rapid and significant downregulation of ET-1 mRNA and peptide release in bovine aortic endothelial cells upon hemodynamic shear stress was found, ${ }^{12}$ but no in vivo evidence has been shown.

Drag-reducing polymers (DRPs) are long-chain, bloodsoluble macromolecules, which have been shown to greatly reduce frictional resistance in flow. ${ }^{13}$ Previous studies have demonstrated that intravenous injection of even nanomolar concentration of DRPs increased hemodynamic shear stress $^{14,15}$ and decreased peripheral vascular resistance. ${ }^{16}$ In this study, we hypothesized that intravenous infusion of DRPs may improve left ventricular hypertrophy and aortic remodeling in spontaneously hypertensive rats (SHR) through increasing blood shear stress.

\section{Materials and methods Preparation of DRPs}

DRP solution was prepared by polyethylene oxide (PEO; Sigma-Aldrich Co., St Louis, MO, USA), with an average molecular weight (MW) of $5 \times 10^{6} \mathrm{Da}$. The polymer was carefully dissolved in normal saline (NS) at a concentration of 1,000 ppm, avoiding mechanical shear degradation of the long-chain macromolecules, and then dialyzed against NS for 24 hours using a membrane (Regenerated Cellulose dialysis membrane, Spectra; Spectrum Laboratories Inc., NJ, USA) with 50-kDa MW cutoff. The stock PEO solution of $1,000 \mathrm{ppm}$ concentration was diluted to a concentration of $20 \mathrm{ppm}$ or $10 \mathrm{ppm}$ with saline and mixed for 1 hour on a slow rocker prior to use.

\section{Animals and procedures}

All experimental procedures in this study were approved by the Animal Care and Use Committee of the Southern Medical University (Guangzhou, Guangdong, People's Republic of China). The investigation conformed to the Guide for the Care and Use of Laboratory Animals published by the US National Institutes of Health (NIH Publication updated in 2011). In all, 24 male SHR (8-week old, 180-220 g, provided by the Vital River) were randomly divided into three groups: NS group ( $\mathrm{n}=8), 10 \mathrm{ppm}$ group $(\mathrm{n}=8)$ and $20 \mathrm{ppm}$ group $(n=8)$. One control group of age-matched male normotensive Wistar rats (WR) received NS. A syringe pump (TE311; Terumo Corporation, Tokyo, Japan) was utilized to guarantee a constant-rate infusion at $3 \mathrm{~mL} / \mathrm{h}$ for $30 \mathrm{~min}$ via the rat tail vein every 2 days for 2 months. Rats were housed at constant room temperature, humidity and light cycles (12/12 h light-dark); had free access to tap water and were fed standard chow ad libitum. At 8 weeks with this treatment and after echocardiography measurements, animals were weighed and sacrificed with a lethal dose of sodium pentobarbital (60 mg/kg i.p.); blood samples were collected for the enzyme-linked immunosorbent assay (ELISA). Then, the heart was removed and the left ventricle (LV) was dissected. Some parts of LV tissue were snap frozen in liquid nitrogen and stored at $-80^{\circ} \mathrm{C}$ for subsequent quantitative polymerase chain reaction (qPCR). The remaining tissues were fixed in $10 \%$ formalin, and paraffin was embedded for histological assessment.

\section{DRP treatments}

Sixteen male WR (250-300 g) were randomly and evenly divided into two groups, a DRP of 10 ppm group (WR +10 ) and a saline group (WR $+\mathrm{NS}$ ). The rats were anesthetized by intraperitoneal injection of pentobarbital sodium $(45 \mathrm{mg} / \mathrm{kg})$. After opening the abdominal cavity, an ultrasonic flow probe (Transonics Inc., Park City, UT, USA) was placed around the abdominal aorta $5 \mathrm{~mm}$ above the common iliac artery. NS or 10 ppm PEO was injected via the caudal vein at a constant rate of $5 \mathrm{~mL} / \mathrm{h}$ for $20 \mathrm{~min}$ according to the assigned treatment group. Shear stress was calculated by the following equation:

$$
\tau=\frac{32 \mu Q}{\pi d^{3}}
$$

where $\mu$ is the blood viscosity, $Q$ is the flow velocity and $d$ is the diameter of abdominal aorta.

\section{Measurement of body weight (BW), heart rate $(\mathrm{HR})$ and systolic blood pressure (SBP)}

BW, HR and SBP were measured at the beginning, 20th, 40th and 60th days of the experiment when all animals were in conscious conditions. SBP was measured in all rats using tail-cuff method. ${ }^{17}$ Ambient temperature was maintained at $30^{\circ} \mathrm{C}$. Before the measurement, the rats were trained to adapt themselves to the restraining cages and tail-cuff apparatus for the standard non-invasive tail-cuff. 


\section{Echocardiography measurement}

Transthoracic echocardiography was performed with the use of a high-resolution echocardiographic system (Sequoia 512; Acuson, Siemens, Germany) equipped with a 14-MHz linear transducer (17L5 probe; Acuson). The transducer was located overlying the chest to produce the optimal parasternal short axis two-dimensional images. A parasternal short-axis M-mode image at the papillary muscle level was acquired to measure the left ventricular end-systolic diameter (LVESD) and left ventricular end-diastolic diameter (LVEDD), left ventricular end-systolic posterior wall thickness (LVPWS) and left ventricular end-systolic posterior wall-depth (LVPWD). Left ventricular systolic function was also assessed from these measurements by calculating the left ventricle fractional shortening (LVFS) and the left ventricle ejection fraction (LVEF). LVFS and LVEF were calculated as follows:

$$
\text { LVFS }=\frac{\text { LVEDD }- \text { LVESD }}{\text { LVEDD }} \times 100 \%
$$

LVEF $=$

$\frac{\mathrm{LV} \text { end-diastolic volume }-\mathrm{LV} \text { end-systolic volume }}{\text { LV end-diastolic volume }} \times 100 \%$

The LV volume was calculated by the formula of Teichholz:

$$
V=[7 /(2.4+D)] \times D^{3}
$$

where $V$ is the $\mathrm{LV}$ volume and $D$ is the $\mathrm{LV}$ dimension. ${ }^{18}$

\section{Measurement of plasma and myocardial endothelin (ET) level}

Both plasma and homogenated myocardial ET levels were measured by using an ELISA kit (Biocalvin, Catalog No EIA-3600, Shanghai, People's Republic of China) according to the manufacturer's instructions.

\section{Histological assessment}

LV and thoracic aorta were fixed in $10 \%$ formalin, dehydrated, and then embedded in paraffin. Subsequently, $4 \mu \mathrm{m}$ thick sections were cut and stained with hematoxylin and eosin or Masson's trichrome. The cross-sectional areas (CSAs) of cardiomyocytes and collagen volume fraction in left ventricle (LVCVF) were quantified from each group. The medial thickness of thoracic aorta was quantified from each group. All image analysis was performed in a blind manner using Image-Pro Plus (version 6.0; Media Cybernetics, Rockville, MD, USA).

\section{RNA extraction and quantitative reverse transcription polymerase chain reaction (qRT-PCR) analysis}

Total RNA was isolated from snap-frozen LV samples with RNAiso Plus (TaKaRa, Dalian, People's Republic of China), and RNA was subjected to reverse transcription reaction using the PrimeScript reverse transcription reagent kit (DRR037A; TaKaRa) according to the manufacturer's protocols. Quantitative analysis of the change in expression levels was performed using SYBR Premix Ex Taq(DRR041A; TaKaRa) at ABI 7500 (Thermo Fisher Scientific, Waltham, MA, USA). Primers for ET-1 and $\beta$-actin were constructed with the help of ShengGong Bio-Tech Co. (Shanghai, People's Republic of China). The primer sequences for polymerase chain reaction (PCR) were as follows: ET-1, 5'-CTGGACATCATCTGGGTCAA-3' and 5'-CTGTTCCCTTGGTCTGTGGT-3', 122 bp and $\beta$-actin, 5'-TGTCACCAACTGGGACGATA-3' and $5^{\prime}$-GGGG TGTTGAAGGTCTCAAA-3', 165 bp.

The alteration in mRNA expression in rats treated with DRPs or NS was assessed by the $2^{-\Delta \Delta C_{t}}$ method.

\section{Statistical analysis}

Values are expressed as mean \pm standard error of the mean (SEM). Statistical significance between multiple groups was determined by using one-way analysis of variance (ANOVA), followed by the Bonferroni's multiple comparison test for comparisons between groups. $P<0.05$ was considered to be a statistically significant difference.

\section{Results BW, HR and SBPs}

During 60 days of treatment, BW in SHR and WR groups ( $\mathrm{n}=8$ per group) increased over time, and there were no significant differences (data not shown). SBP in the WR + NS group was significantly lower than SBP in all SHR groups ( $\mathrm{n}=8$ per group; Figure 1), while there was no significant difference between the SHR + NS, SHR + 10DRP and SHR + 20DRP groups. HR did not differ among all groups (data not shown).

\section{DRPs improve shear stress of abdominal aortic flow}

Abdominal aortic flow was significantly higher in the WR group treated with $10 \mathrm{ppm}$ PEO compared to the saline-treated group ( $\mathrm{n}=8$ per group, $P<0.05$; Figure $2 \mathrm{~A}$ ). What is more, the change of shear stress in abdominal aorta was markedly higher 


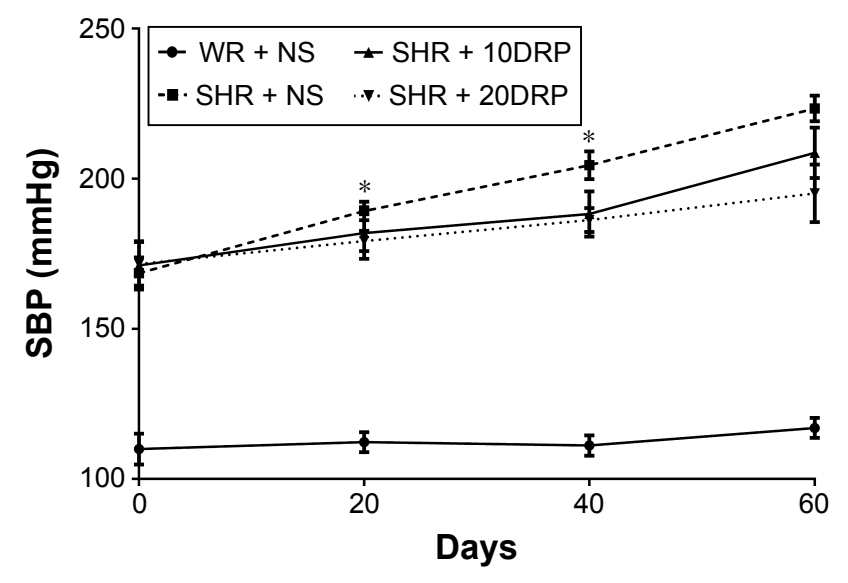

Figure I Comparison of SBP at different time points in each group (mean \pm SEM, $\mathrm{n}=8$ per group).

Note: $* P<0.05$ compared with the WR + NS group.

Abbreviations: SBP, systolic blood pressure; SEM, standard error of the mean; WR, Wistar rats; NS, normal saline; SHR, spontaneously hypertensive rats.

in the WR group treated with 10 ppm PEO compared to the saline-treated group ( $\mathrm{n}=8$ per group, $P<0.05$; Figure $2 \mathrm{~B}$ ).

\section{DRPs improve left ventricular posterior wall hypertrophy}

After 60 days treatment, M-mode echocardiography was conducted in vitro to assess myocardial hypertrophy ( $n=8$ per group; Figure 3A). The results showed significant increase in LVPWS (3.31 \pm 0.21 vs $2.62 \pm 0.11$, respectively; $P=0.015$; $\mathrm{n}=8$ per group; Figure 3$)$ and LVPWD $(2.58 \pm 0.05$ vs $1.91 \pm 0.11$, respectively; $P=0.002 ; n=8$ per group; Figure $3 \mathrm{E}$ ) in the SHR + NS group compared with the WR + NS group. However after DRP administration, the LVPWS of rats in the SHR + 10DRP and SHR + 20DRP groups was significantly decreased compared with that of rats in the SHR + NS group (2.65 \pm 0.12 and $2.63 \pm 0.14$ vs $3.31 \pm 0.21, P=0.002$ and $P=0.017$, respectively; $n=8$ per group; Figure $3 \mathrm{D}$ ). What is more, the LVPWD of rats in the SHR + 20DRP group was significantly decreased compared with that of rats in the SHR + NS group (2.03 \pm 0.14 vs $2.58 \pm 0.05$, respectively; $P=0.011 ; n=8$ per group; Figure 3E). Besides, LVESD and LVEDD of rats in the SHR + 10DRP and SHR + 20DRP groups were significantly increased compared with those of rats in the SHR + NS group ( $n=8$ per group; Figure $3 \mathrm{~B}$ and $\mathrm{C}$ ), while there was no significant difference in fractional shortening (FS) and ejection fraction (EF; $n=8$ per group; Figure $3 F$ and $G$ ).

\section{DRPs prevent left ventricular hypertrophy and myocardium fibrosis}

As shown in Figure $4 \mathrm{~A}$ and $\mathrm{B}$, there was a significant increase in CSA in the SHR + NS group compared with the
WR + NS group $\left(630.97 \pm 54.15 \mu \mathrm{m}^{2}\right.$ vs $309.34 \pm 9.91 \mu \mathrm{m}^{2}$, respectively; $P=0.032 ; \mathrm{n}=3$ per group; Figure $4 \mathrm{~A}$ and $\mathrm{B}$ ). These changes were attenuated after DRPs treatment, as the CSA of the SHR + 20DRP group was significantly suppressed $\left(291.72 \pm 29.08 \mu \mathrm{m}^{2}\right.$ vs $630.97 \pm 54.15 \mu \mathrm{m}^{2}$, respectively; $P=0.016 ; \mathrm{n}=3$ per group; Figure $4 \mathrm{~A}$ and $\mathrm{B}$ ). Masson's trichrome staining clearly showed increased LVCVF in the $\mathrm{SHR}+\mathrm{NS}$ group compared with the WR + NS group (6.58\% $\pm 0.49 \%$ vs $1.86 \% \pm 0.37 \%$, respectively; $P=0.0002$; $\mathrm{n}=3$ per group; Figure $4 \mathrm{C}$ and $\mathrm{D})$. These changes were significantly reversed in the SHR + 10DRP and SHR + 20DRP groups $(2.70 \% \pm 0.34 \%, 2.38 \% \pm 0.34 \%$ vs $6.58 \% \pm 0.49 \%$; $P=0.001$ and $P=0.0004$, respectively; $n=3$ per group; Figure $4 \mathrm{C}$ and D), but without significant differences between them ( $n=3$ per group; Figure $4 C$ and D).

\section{DRPs improve aortic medial thickness}

As shown in Figure 5, the medial thickness of aorta in the SHR + NS group was significantly higher than that in the $\mathrm{WR}+\mathrm{NS}$ group $(164.97 \pm 3.37 \mu \mathrm{m}$ vs $126.63 \pm 4.01 \mu \mathrm{m}$, respectively; $P=0.002$; $\mathrm{n}=4$ per group; Figure $5 \mathrm{~A}$ and $\mathrm{B}$ ). The treatment with DRPs significantly reduced the medial thickness in the SHR + 10DRP and SHR + 20DRP groups (132.73 $\pm 7.12 \mu \mathrm{m}, 135.46 \pm 6.79 \mu \mathrm{m}$ vs $164.97 \pm 3.37 \mu \mathrm{m}$; $P=0.009$ and $P=0.017$, respectively; $\mathrm{n}=4$ per group; Figure $5 \mathrm{~A}$ and $\mathrm{B}$ ).

\section{DRPs attenuate ET-I expression in LV and aorta}

Immunohistochemistry clearly showed increased expression of ET-1 protein in the cardiomyocytes and aorta of rats in the SHR + NS group compared with in the WR + NS group, which was attenuated by DRP administration in the SHR + 10DRP and SHR + 20DRP groups ( $n=3$ per group; Figure 6A and B). Real-time PCR showed that ET-1 gene expression was markedly decreased in the SHR + 20DRP group compared with in the SHR + NS group (1.46 \pm 0.14 vs $2.24 \pm 0.07$, respectively; $P=0.001 ; n=3$ per group; Figure $6 \mathrm{C}$ ). These results were in parallel with the left ventricular tissue and serum ET levels ( $n=8$ per group; Figure 6D and E).

\section{Discussion}

Although DRPs have shown potent hydrodynamic effects to increase blood flow and decrease vascular resistance, it is still unknown whether increased blood flow would affect ET-1 expression and cardiac/aortic remodeling. In this study, we assessed the effect of DRPs in the process of cardiac and aortic remodeling caused by hypertension and demonstrated that blood shear stress and ET-1 expression may play 

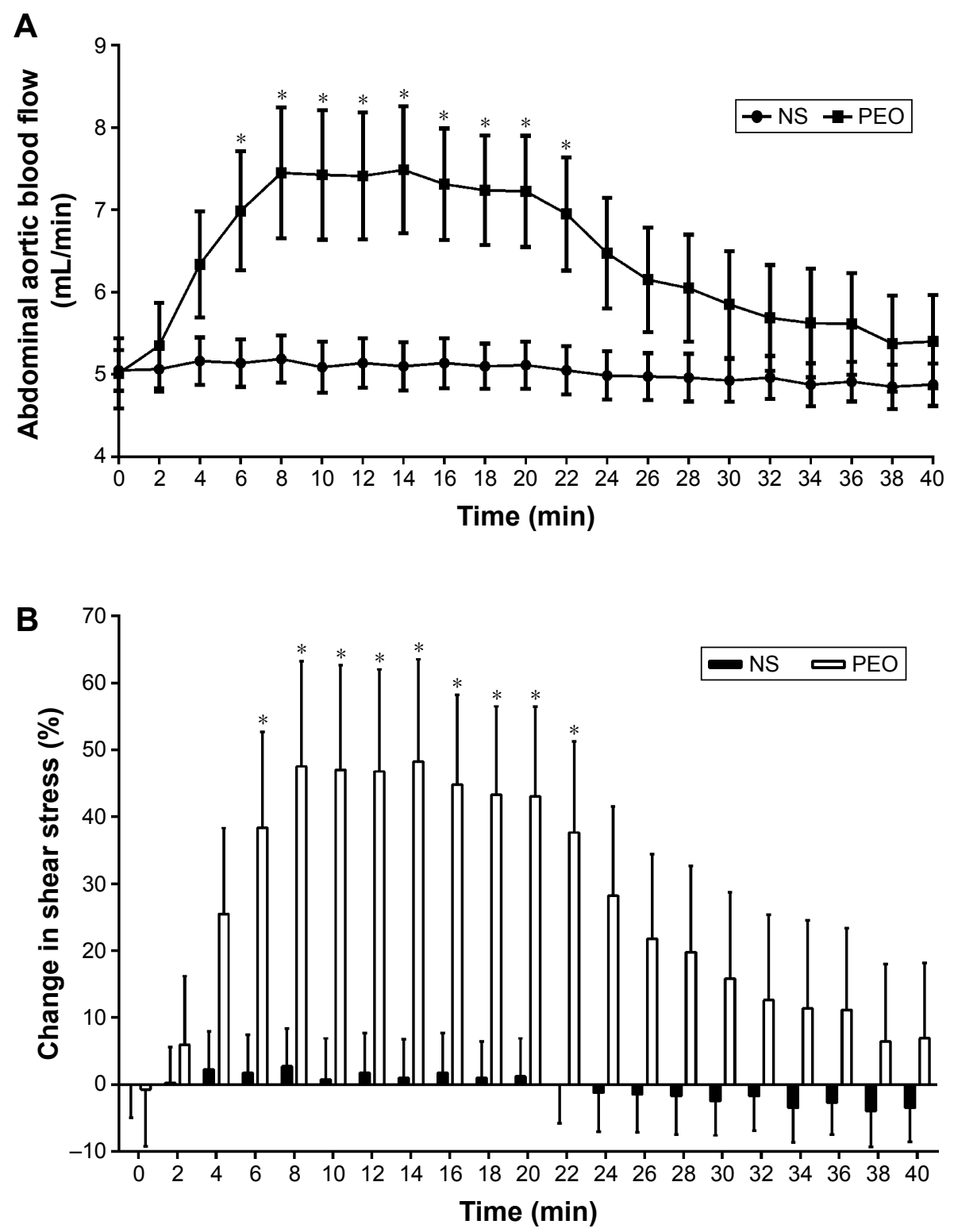

Figure 2 DRPs improve abdominal aortic blood flow and shear stress in SHR.

Notes: (A) After treatment with NS or PEO in SHR for 20 min, abdominal aortic blood flow was measured by echocardiography. PEO significantly increased blood flow compared with NS. (B) Shear stress was calculated by $\tau=32 \mu Q / \pi d^{3}$, where $\mu$ is the blood viscosity, $Q$ is the flow velocity and $d$ is the diameter of abdominal aorta. Mean \pm SEM, $n=8$ per group and $* P<0.05$ compared with NS.

Abbreviations: DRP, drag-reducing polymer; SHR, spontaneously hypertensive rats; NS, normal saline; PEO, polyethylene oxide; SEM, standard error of the mean.

important roles in cardiac/aortic remodeling. To our best knowledge, this is the first report showing chronic administration of DRPs into the systemic circulation improves the cardiac and aortic remodeling, by increasing blood shear stress and suppressing the ET-1 expression.

DRPs are long-chain, blood soluble macromolecules, which have been shown to greatly reduce frictional resistance in both turbulent and laminar flow, known as Toms effect. ${ }^{13,19}$ DRPs were initially used in fire fighting, irrigation and petroleum pumping to reduce frictional energy losses in large pipes. Recently, these observations have been extended to the vascular system and some biological effects of DRPs have been reported. It was found that acute administration of nanomolar concentration of DRPs improved LV function in the rat model of myocardial infarction. ${ }^{20}$ DRPs also enhanced microvascular perfusion in vivo. In a canine model of flowlimiting coronary artery stenosis, DRPs improved perfusion to myocardium by decreasing microvascular resistance through an increase in capillary volume and red blood cell (RBC) velocity. ${ }^{21}$ In our study, DRPs significantly increased blood flow and blood shear stress (Figure 2).

The family of ETs consists of four closely related peptides - ET-1, ET-2, ET-3 and ET-4 - which originate from large preproendothelin peptides and are synthesized 


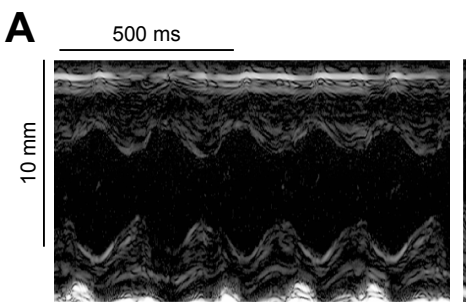

WR + NS

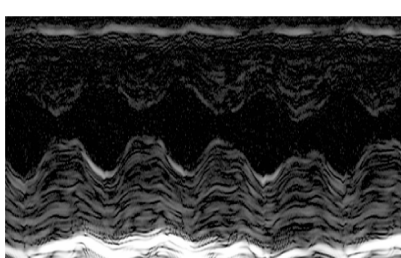

SHR + NS

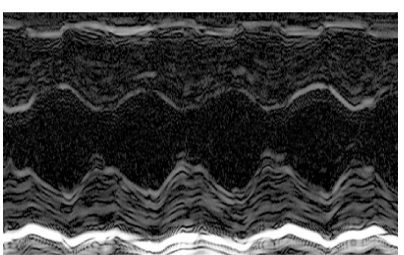

SHR + 10DRP

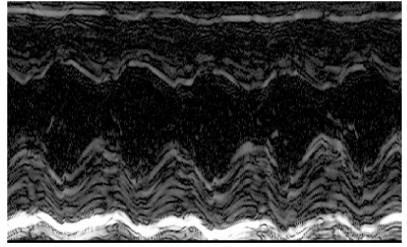

SHR + 20DRP
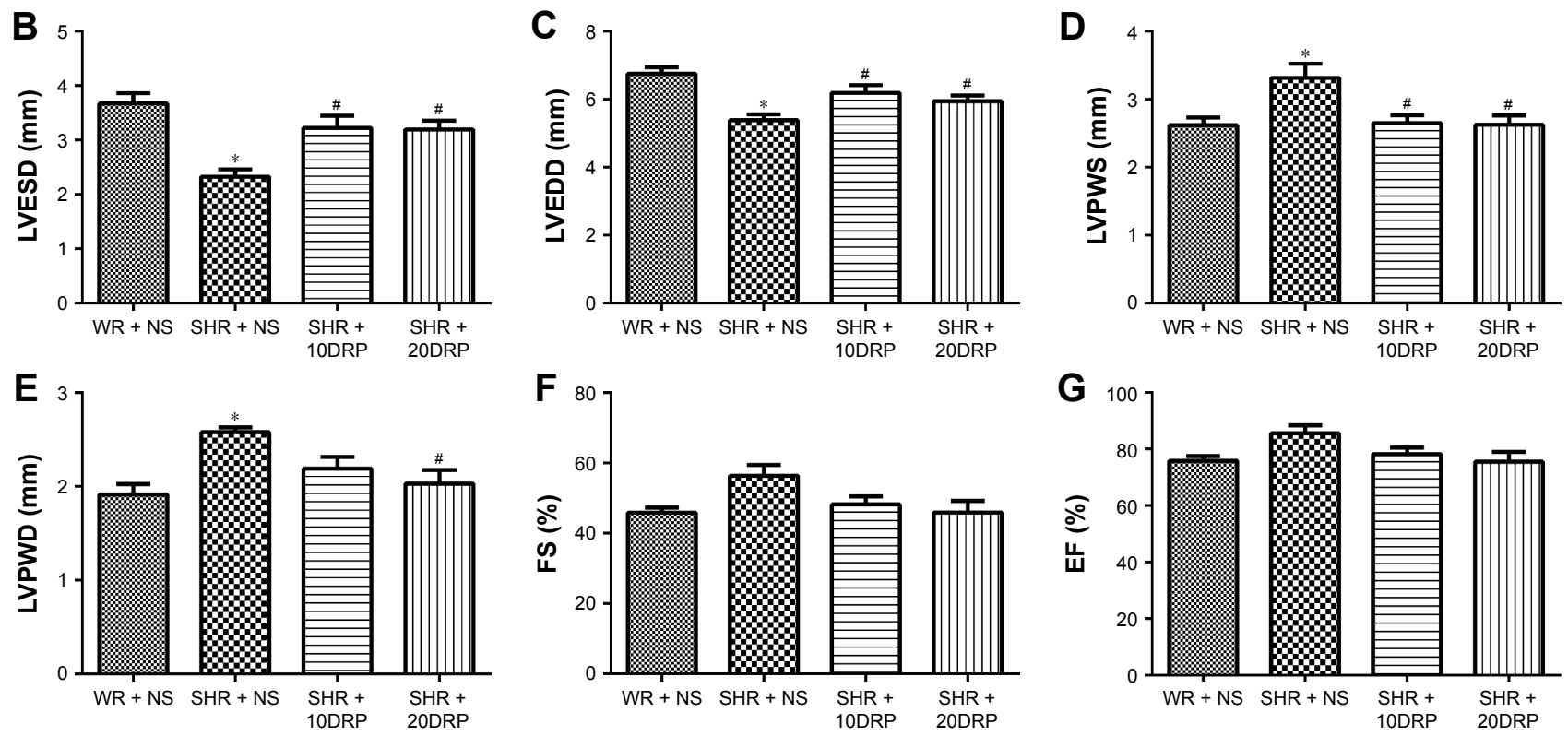

Figure 3 DRPs improve cardiac hypertrophy in SHR.

Notes: (A) Representative images of M-mode echocardiography obtained with two-dimensional guidance from a short-axis midventricular view. (B) LVESD, (C) LVEDD, (D) LVPWS, (E) LVPWD, (F) LVFS and (G) LVEF are presented as mean \pm SEM. $n=8$ per group. *P<0.05 compared with WR + NS and ${ }^{*} P<0.05$ compared with SHR + NS. Abbreviations: DRP, drag-reducing polymer; SHR, spontaneously hypertensive rats; LVESD, left ventricular end-systolic diameter; LVEDD, left ventricular end-diastolic diameter; LVPWS, left ventricular end-systolic posterior wall thickness; LVPWD, left ventricular end-systolic posterior wall-depth; LVFS, left ventricle fractional shortening; LVEF, left ventricle ejection fraction; SEM, standard error of the mean; WR, Wistar rats; NS, normal saline; FS, fractional shortening; EF, ejection fraction.

by endothelial and smooth muscle cells, as well as neural, renal, pulmonal and inflammatory cells. ${ }^{22}$ ET-1 promotes vasoconstriction and cell proliferation. ${ }^{23}$ The factors modulating the expression of ET-1 are shear stress, pulsatile stretch, epinephrine, angiotensin II and inflammatory cytokines. Shear stress induces rapid and significant downregulation of ET-1 mRNA expression and peptide release with respect to static conditions in bovine aortic endothelial cells and human umbilical vein endothelial cells..$^{24,25}$

ET-1 plays an important role in hypertension. Besides its blood pressure raising effect in human beings, ET-1 induces vascular and myocardial hypertrophies, which are independent risk factors for cardiovascular morbidity and mortality ${ }^{26,27}$ In experimental hypertension, vascular ET-1 content is much more enriched than is plasma, which promotes vasoconstriction and smooth muscle cell proliferation via ETA receptors. ${ }^{28}$ As we can see from Figure 5, ET-1 expression in left ventricular and thoracic aorta of the
SHR + NS group was significantly higher compared with that of the WR + NS group. Moreover, in experimental hypertension, treatment with a selective ETA-receptor antagonist attenuated left ventricular hypertrophy, prevented vascular hypertrophy and ameliorated endothelial dysfunction. ${ }^{29}$ In our study, intravenous infusion of DRPs significantly increased blood shear stress, which may suppress ET-1 expression in left ventricular and thoracic aorta and left ventricular hypertrophy and aortic remodeling (Figures 3-5).

It should be noted that the sample size in this study is relatively small with some significant variation in some of the data, and this would inevitably weaken the results and might be the reason why no difference is seen between the two groups (SHR + 10DRP and SHR + 20DRP).

\section{Conclusion}

This work demonstrated for the first time that chronic intravenous infusion of DRPs significantly improved cardiac and 
A
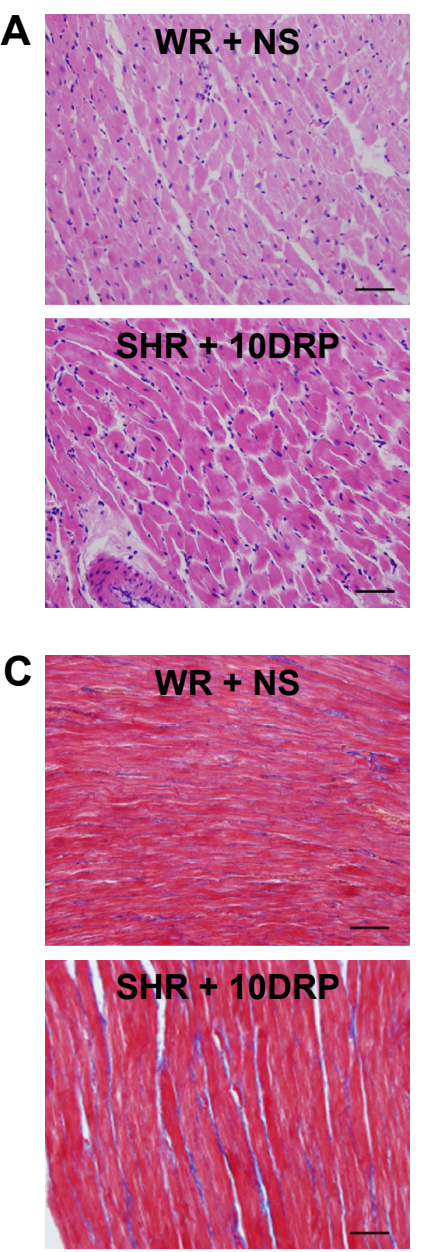
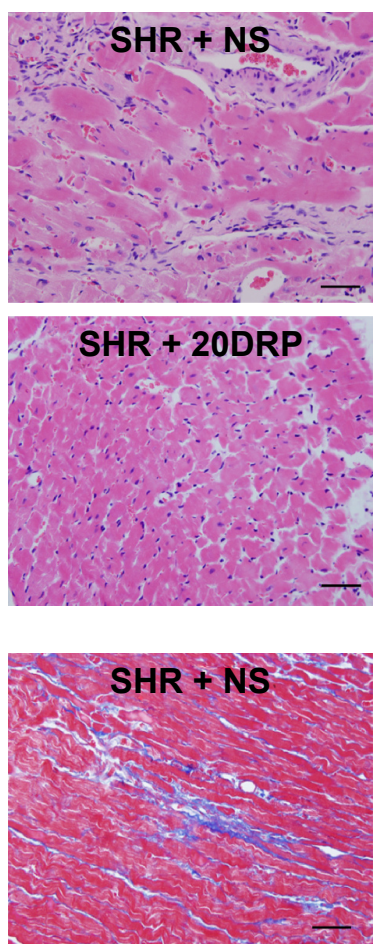

SHR + 20DRP

Figure 4 DRPs improve left ventricular hypertrophy and myocardial fibrosis.

B

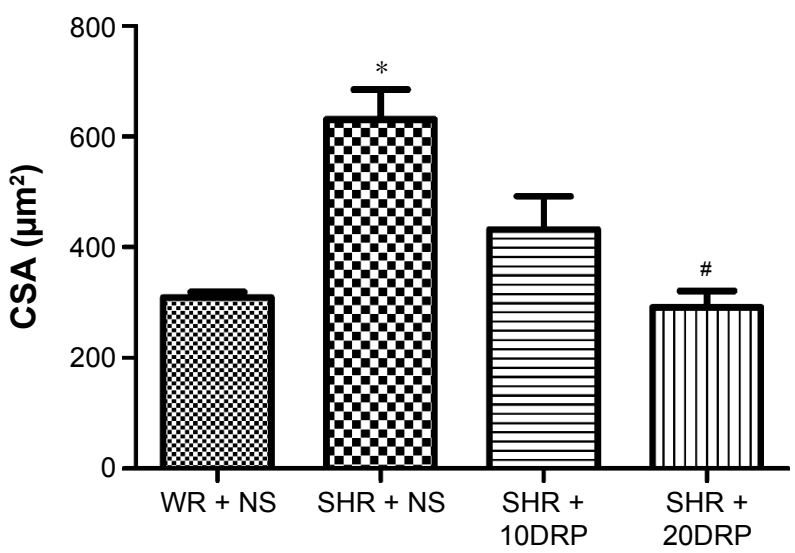

D

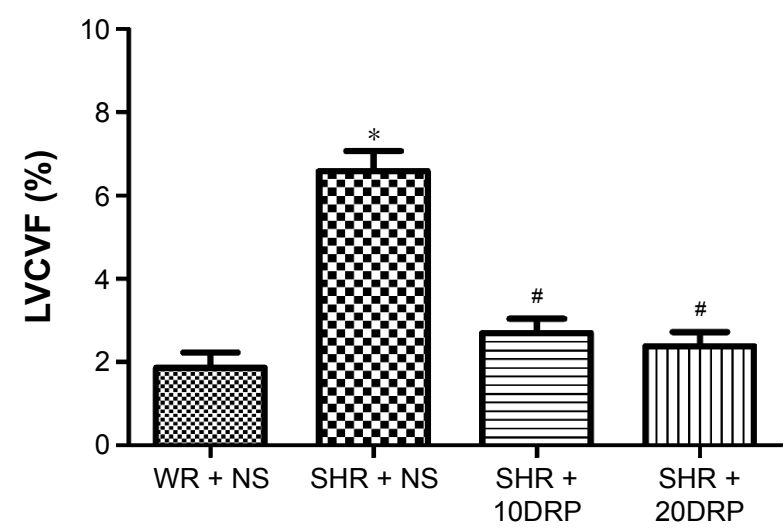

Notes: (A) Representative micrographs of cross sections of LV cardiomyocytes of each group. Scale bars: $50 \mu \mathrm{m}$. (B) Quantitative analysis of the CSA of LV cardiomyocytes. (C) Representative images of LV with Masson's trichrome stain of each group. (D) Quantitative analysis of LVCVF. Values are mean \pm SEM, $n=3$ per group, $* P<0.05$ compared with WR + NS and ${ }^{\# P}<0.05$ compared with SHR + NS. Scale bars: $50 \mu \mathrm{m}$.

Abbreviations: DRP, drag-reducing polymer; LV, left ventricle; CSA, cross-sectional area; LVCVF, collagen volume fraction in left ventricle; SEM, standard error of the mean; WR, Wistar rats; NS, normal saline; SHR, spontaneously hypertensive rats.

A
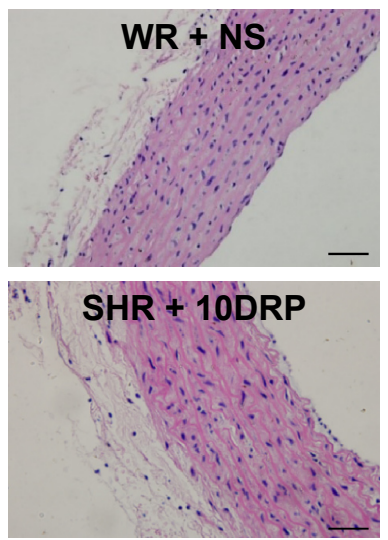

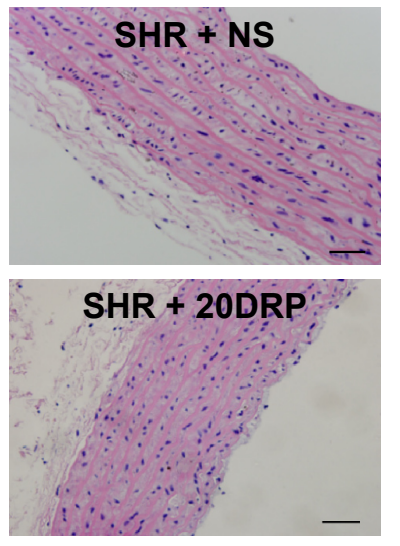

B

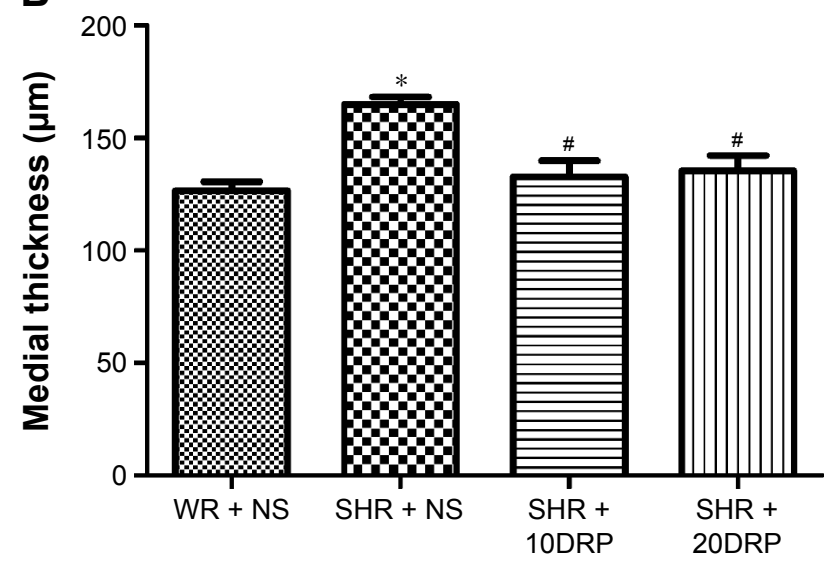

Figure 5 DRPs reduce aortic medial thickness in SHR.

Notes: (A) Representative images of thoracic aorta with hematoxylin and eosin staining of each group. Scale bar: $50 \mu \mathrm{m}$. (B) Quantitative analysis of medial thickness ( $\mu \mathrm{m}$ ). Values are mean $\pm S E M, n=4$ per group, ${ }^{* P}<0.05$ compared with $W R+N S$ and ${ }^{\#} P<0.05$ compared with $S H R+N S$.

Abbreviations: DRP, drag-reducing polymer; SHR, spontaneously hypertensive rats; SEM, standard error of the mean; WR, Wistar rats; NS, normal saline. 
A
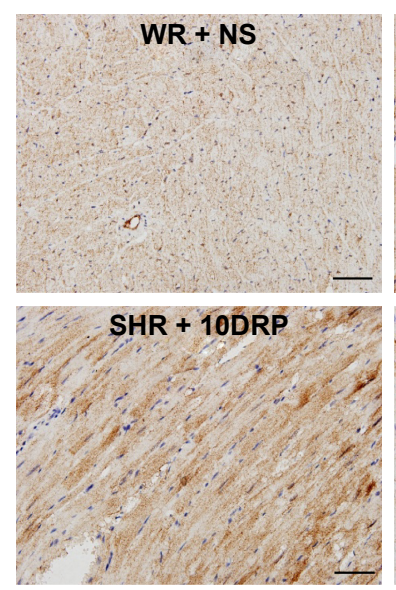
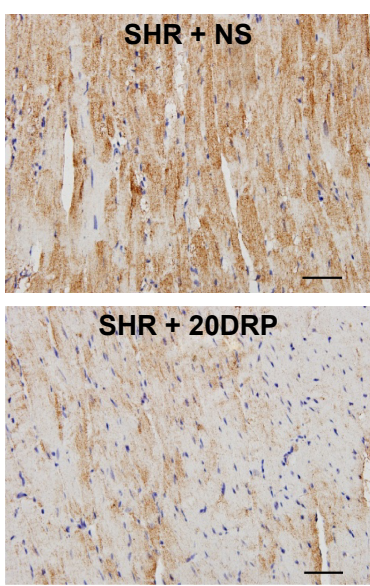

B
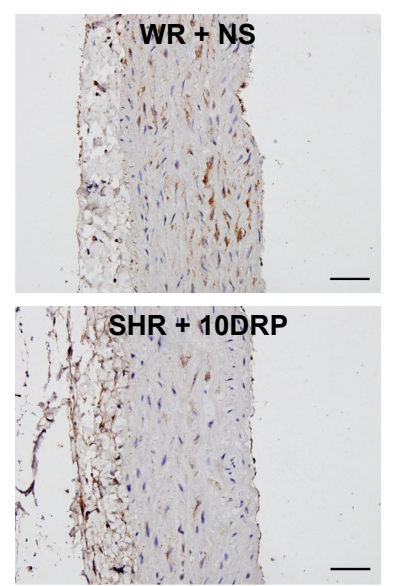

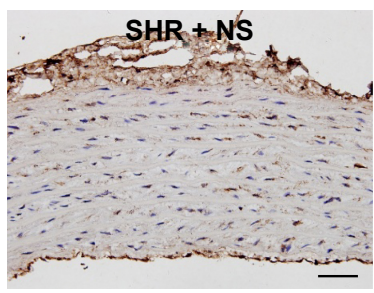

SHR + 20DRP

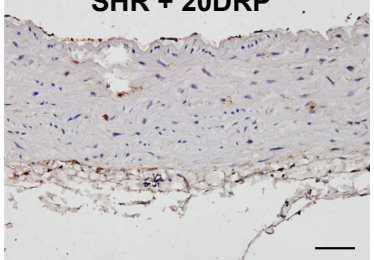

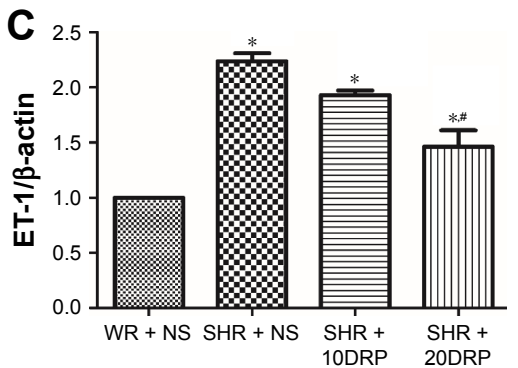
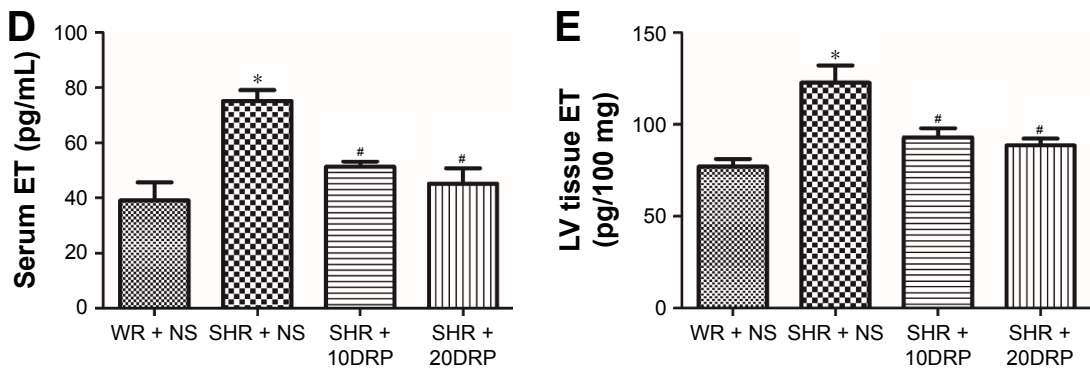

Figure 6 DRPs attenuate ET-I expression in LV and aorta.

Notes: Immunohistochemical detection of ET-I expression in LV (A) and thoracic aorta (B). Scale bar: $50 \mu$ m. (C) Left ventricular ET-I mRNA levels in each group ( $\mathrm{n}=3$ per group). (D) Serum ET levels and (E) left ventricular tissue ET levels of each group ( $n=8$ per group). Values are mean \pm SEM, $* P<0.05$ compared with WR + NS and ${ }^{\#} P<0.05$ compared with SHR + NS.

Abbreviations: DRP, drag-reducing polymer; ET-I, endothelin-I; LV, left ventricle; ET, endothelin; SEM, standard error of the mean; WR, Wistar rats; NS, normal saline; SHR, spontaneously hypertensive rats.

aortic remodeling in SHR through increasing blood shear stress and attenuating ET-1 expression.

\section{Acknowledgments}

We thank Cardiology Lab and Medical Research Center of Nanfang Hospital for their critical comments and technical support. This project was supported by the National Natural Science Foundation of China (81470598, 81370235, 81170101 ) and the Natural Science Foundation of Guangdong Province of China (S2012010009024). The sponsors had no role in study design, data collection and analysis, our decision to publish, or the preparation of the manuscript.

\section{Disclosure}

The authors report no conflicts of interest in this work.

\section{References}

1. Cohn JN, Ferrari R, Sharpe N. Cardiac remodeling - concepts and clinical implications: a consensus paper from an international forum on cardiac remodeling. Behalf of an International Forum on Cardiac Remodeling. J Am Coll Cardiol. 2000;35(3):569-582.

2. Arribas SM, Hinek A, Gonzalez MC. Elastic fibres and vascular structure in hypertension. Pharmacol Ther. 2006;111(3):771-791.

3. Lujan HL, Janbaih H, Dicarlo SE. Structural remodeling of the heart and its premotor cardioinhibitory vagal neurons following T5 spinal cord transection. J Appl Physiol (1985). 2014;116(9):1148-1155.
4. Cohn JN. Left ventricle and arteries: structure, function, hormones, and disease. Hypertension. 2001;37(2 pt 2):346-349.

5. Li X, Han J, Li L, Wang KJ, Hu SJ. Effect of farnesyltransferase inhibition on cardiac remodeling in spontaneously hypertensive rats. Int $J$ Cardiol. 2013;168(4):3340-3347.

6. Guerrero EI, Ardanaz N, Sevilla MA, Arevalo MA, Montero MJ. Cardiovascular effects of nebivolol in spontaneously hypertensive rats persist after treatment withdrawal. J Hypertens. 2006;24(1):151-158.

7. Gomez-Roso M, Montero MJ, Carron R, Sevilla MA. Cardiovascular changes in spontaneously hypertensive rats are improved by chronic treatment with zofenopril. Br J Pharmacol. 2009;158(8):1911-1921.

8. Amiri F, Virdis A, Neves MF, et al. Endothelium-restricted overexpression of human endothelin-1 causes vascular remodeling and endothelial dysfunction. Circulation. 2004;110(15):2233-2240.

9. Thandapilly SJ, Louis XL, Behbahani J, et al. Reduced hemodynamic load aids low-dose resveratrol in reversing cardiovascular defects in hypertensive rats. Hypertens Res. 2013;36(10):866-872.

10. Topper JN, Gimbrone MA Jr. Blood flow and vascular gene expression: fluid shear stress as a modulator of endothelial phenotype. Mol Med Today. 1999;5(1):40-46.

11. Hahn C, Schwartz MA. Mechanotransduction in vascular physiology and atherogenesis. Nat Rev Mol Cell Biol. 2009;10(1):53-62.

12. Malek AM, Greene AL, Izumo S. Regulation of endothelin 1 gene by fluid shear stress is transcriptionally mediated and independent of protein kinase C and cAMP. Proc Natl Acad Sci U S A. 1993;90(13): 5999-6003.

13. Bhattacharjee JK, Thirumalai D. Drag reduction in turbulent flows by polymers. Phys Rev Lett. 1991;67(2):196-199.

14. Sawchuk AP, Unthank JL, Dalsing MC. Drag reducing polymers may decrease atherosclerosis by increasing shear in areas normally exposed to low shear stress. J Vasc Surg. 1999;30(4):761-764. 
15. Faruqui FI, Otten MD, Polimeni PI. Protection against atherogenesis with the polymer drag-reducing agent Separan AP-30. Circulation. 1987; 75(3):627-635.

16. Polimeni PI, Ottenbreit BT. Hemodynamic effects of a poly(ethylene oxide) drag-reducing polymer, Polyox WSR N-60K, in the open-chest rat. J Cardiovasc Pharmacol. 1989;14(3):374-380.

17. Widdop RE, Li XC. A simple versatile method for measuring tail cuff systolic blood pressure in conscious rats. Clin Sci (Lond). 1997; 93(3):191-194.

18. Teichholz LE, Kreulen T, Herman MV, Gorlin R. Problems in echocardiographic volume determinations: echocardiographic-angiographic correlations in the presence of absence of asynergy. Am J Cardiol. 1976; 37(1):7-11.

19. Brands J, Kliner D, Lipowsky HH, Kameneva MV, Villanueva FS, Pacella JJ. New insights into the microvascular mechanisms of drag reducing polymers: effect on the cell-free layer. PLoS One. 2013;8(10): e77252.

20. Chen X, Zha D, Xiu J, et al. A new hydrodynamic approach by infusion of drag-reducing polymers to improve left ventricular function in rats with myocardial infarction. Int J Cardiol. 2011;147(1):112-117.

21. Pacella JJ, Kameneva MV, Csikari M, Lu E, Villanueva FS. A novel hydrodynamic approach to the treatment of coronary artery disease. Eur Heart J. 2006;27(19):2362-2369.

22. Ikegawa R, Matsumura Y, Tsukahara Y, Takaoka M, Morimoto S. Phosphoramidon, a metalloproteinase inhibitor, suppresses the secretion of endothelin-1 from cultured endothelial cells by inhibiting a big endothelin-1 converting enzyme. Biochem Biophys Res Commun. 1990; 171(2):669-675
23. Seo B, Oemar BS, Siebenmann R, von Segesser L, Luscher TF. Both ETA and ETB receptors mediate contraction to endothelin-1 in human blood vessels. Circulation. 1994;89(3):1203-1208.

24. Malek AM, Zhang J, Jiang J, Alper SL, Izumo S. Endothelin-1 gene suppression by shear stress: pharmacological evaluation of the role of tyrosine kinase, intracellular calcium, cytoskeleton, and mechanosensitive channels. J Mol Cell Cardiol. 1999;31(2):387-399.

25. Vozzi F, Bianchi F, Ahluwalia A, Domenici C. Hydrostatic pressure and shear stress affect endothelin-1 and nitric oxide release by endothelial cells in bioreactors. Biotechnol J. 2014;9(1):146-154.

26. Vierhapper H, Wagner O, Nowotny P, Waldhausl W. Effect of endothelin-1 in man. Circulation. 1990;81(4):1415-1418.

27. Barton M, d'Uscio LV, Shaw S, Meyer P, Moreau P, Luscher TF. ET(A) receptor blockade prevents increased tissue endothelin-1, vascular hypertrophy, and endothelial dysfunction in salt-sensitive hypertension. Hypertension. 1998;31(1 pt 2):499-504.

28. Moreau P, d'Uscio LV, Shaw S, Takase H, Barton M, Luscher TF. Angiotensin II increases tissue endothelin and induces vascular hypertrophy: reversal by ET(A)-receptor antagonist. Circulation. 1997;96(5): 1593-1597.

29. Lee TM, Lin MS, Chou TF, Tsai CH, Chang NC. Effect of pravastatin on development of left ventricular hypertrophy in spontaneously hypertensive rats. Am J Physiol Heart Circ Physiol. 2005;289(1):H220-H227.
International Journal of Nanomedicine

\section{Publish your work in this journal}

The International Journal of Nanomedicine is an international, peerreviewed journal focusing on the application of nanotechnology in diagnostics, therapeutics, and drug delivery systems throughout the biomedical field. This journal is indexed on PubMed Central, MedLine, CAS, SciSearch $®$, Current Contents $\AA /$ Clinical Medicine,

\section{Dovepress}

Journal Citation Reports/Science Edition, EMBase, Scopus and the Elsevier Bibliographic databases. The manuscript management system is completely online and includes a very quick and fair peer-review system, which is all easy to use. Visit http://www.dovepress.com/ testimonials.php to read real quotes from published authors. 